SE; F00-F99) $64.9 \%$ in women and $48.8 \%$ in men, for musculoskeletal disorders (M00-M99) 91.1\% and 90.0\%, respectively.

The prevalence of premature age pension increased between 2004 and 2011: men $2.5 \%$ to $6.4 \%$, women $1.7 \%$ to $5.5 \%$. Blue collar occupations were most affected.

Conclusions The socioeconomic divide in lost years of working life between white and blue collars prevailed. There was an apparent flow from disability to premature age pension, in particular in female blue collars. The findings indicate the budgetary savings of disability pensions transferred the economic burden of disease to individuals, and mainly to female blue collar workers.

\section{D.6 INVENTORY OF OCCUPATIONAL, INDUSTRIAL AND POPULATION COHORTS IN SWITZERLAND}

${ }^{1}$ Nicolas Bovio*, ${ }^{2}$ Danielle Vienneau, ${ }^{1}$ Irina Guseva Canu. ${ }^{1}$ Institut universitaire romand de Santé au Travail (IST), Faculty of Biology and Medecine, University of Lausanne, Lausanne, Switzerland; ' 2 Swiss Tropical and Public Health Institute (Swiss TPH), Department of Epidemiology and Public Health, University of Basel, Basel, Switzerland

\subsection{6/OEM-2019-EPI.77}

Context Given the importance of harmonization in occupational epidemiology (OE) research, an European network, OMEGA-NET, is developing an inventory of occupational, industrial and population cohorts in Europe. We inventorized existing cohorts in Switzerland and assessed their relevance for OE.

Methods We identified cohorts based on the review of data repositories and publications of the leading occupational and public health institutions in Switzerland. Cohorts were considered relevant for $\mathrm{OE}$ if data on occupation were available. The quality of these data was assessed critically.

Results In Switzerland, we found no industrial cohort, one retrospective occupational cohort exposed to magnetic fields [20,141 Swiss Federal Railway workers, cancer morbidity follow-up=1972-2002] and four population-based cohorts relevant for OE: the census-based Swiss National Cohort (SNC) [5.8 million adult residents in Switzerland, mortality by cause follow-up=1990-2014], the Study on Air Pollution And Lung Disease In Adults (SAPALDIA) [ $\mathrm{n}=9,561$, lung function and morbidity follow-up=1991-present], CoLaus $\mid$ PsyCoLaus $[6,700$ 35-75 year-old residents of Lausanne, cardiovascular and mental morbidity follow-up=2003-present], the Swiss Kidney Project on Genes in Hypertension (SKIPOGH) [1134 residents of Lausanne, Geneva and Berne, kidney and metabolic morbidity follow-up =2009-present].

Occupation was coded using the International Standard Classification of Occupations (ISCO) only in the SNC (ISCO68 and ISCO-88) and SAPALDIA (ISCO-88). In SKIPOGH, the Belgian Classification of occupations was used. In CoLaus| PsyCoLaus, occupation remains uncoded. Noteworthy, the percentage of missing occupations is 43\%, non-reported, $65 \%$ and $61 \%$, respectively.

Conclusion Having detailed high-quality data on multiple health outcomes, the identified Swiss cohorts may represent a valuable contribution to OE research. However, in absence of standardisation in collecting and coding of occupational data in these cohorts, their use in OE is still challenging. Planned harmonization efforts in frame of OMEGA-NET will be beneficial for improving the quality of these data and OE research in Switzerland and abroad.

\section{D.7 CONSTANCES: A POPULATION-BASED COHORT FOR} OCCUPATIONAL EPIDEMIOLOGY

${ }^{1,2}$ Marcel Goldberg*, ${ }^{1,3}$ Alexis Descatha, ${ }^{1}$ Annette Leclerc, ${ }^{4}$ Yves Roquelaure, ${ }^{5}$ Jack Siemiatycki, ${ }^{1,2}$ Marie Zins. 'Inserm Ums 11, Villejuif, France; ${ }^{2}$ Paris Descartes University, Paris, France; ${ }^{3}$ Versailles-Saint Quentin University, Versailles, France; ${ }^{4}$ Univ Angers, CHU Angers, Univ Rennes, Inserm, EHESP, Irset - UMR_S1085, ESTER Team, Angers, France; ${ }^{5}$ Social and Preventive Medicine, University of Montréal School of Public Health, Montréal, Canada

\subsection{6/OEM-2019-EPI.78}

The CONSTANCES general-purpose population-based cohort is intended to serve as an epidemiological research infrastructure accessible to the epidemiologic research community. CONSTANCES also provides useful public health information to the public health authorities. CONSTANCES was designed as a randomly selected sample of French adults aged 18-69 years at inception; 200000 subjects will be included over a six-year period. At inclusion, the selected subjects are invited to complete questionnaires, including a lifetime job history, and to attend a Health Screening Centre (HSC) for a comprehensive health examination. A biobank is being set up. The follow-up includes a yearly self-administered questionnaire, and a periodic visit to an HSC. Social and health data are collected from the French national administrative databases. Data collected for participants include social and demographic characteristics, socioeconomic status, life events, and behaviours. Regarding occupational factors, a full job history and a wealth of data on employment and organizational, chemical, biological, biomechanical and psychosocial lifelong exposure are collected at inception and during the follow-up. The health data cover a wide spectrum: self-reported health scales, reported prevalent and incident diseases, long-term chronic diseases and hospitalizations, sick-leaves, handicaps, limitations, disabilities and injuries, healthcare utilization and services provided, and causes of death. To consider non-participation at inclusion and attrition throughout the longitudinal follow-up, a cohort of non-participants was set up and will be followed through the same national databases as participants.

Inclusion began in 2012 and more than 180000 participants were enrolled by July 2018. Several projects on occupational risks are already in progress, and an Occupational Health Users Club was established. This platform and its potential contributions will be described, as well as the means for international investigators to access it.

\section{O3D.9 PSYCHOSOCIAL CONTEXT, SOMATIC COMPLAINTS, WORK ABILITY, AND JOB SATISFACTION IN ANAESTHESIA HEALTH PROFESSIONALS. SETTING UP A PROSPECTIVE COHORT STUDY}

Dragan Mijakoski*, Jordan Minov, Jovanka Karadzinska-Bislimovska, Sasho Stoleski, Aneta Atanasovska, Dragana Bislimovska. Institute of Occupational Health of RM, WHO CC, Skopje, Macedonia

\subsection{6/OEM-2019-EPI.79}

Background Psychosocial context, characterized by the levels of job demands, shift/night work, burnout, and teamwork, as well as presence of certain somatic complaints (chronic respiratory, skin, and musculoskeletal symptoms) and health disorders could affect the work ability and job satisfaction of health professionals (HPs). 
The aim of this prospective cohort study will be to assess changes in self-reported psychosocial factors, work ability, and job satisfaction, as well as to evaluate the incidence of somatic complaints and health disorders in anaesthesia HPs. Additional objective of the study will be to evaluate associations between analysed variables.

Cohort participants HPs working in a health institution for anaesthesia, reanimation and intensive care from Skopje will be included in the first phase of the study with a possibility to enrol other health institutions during the next phases of the research. The first phase will involve about 120 anaesthesia HPs (about 50 physicians and 70 nurses).

Data collection phases At enrolment point, the participants will be invited to complete questionnaires and to attend a preventive medical examination by a specialist in occupational medicine (OM). The follow-up will include an annually selfadministered questionnaire as well as periodic visits to an OM specialist.

Main types of data collected Data collected for participants will include demographic and job characteristics; physical, organisational, emotional, and cognitive job demands; characteristics of shift/night work; burnout dimensions; teamwork; presence of chronic respiratory, skin, and musculoskeletal symptoms; work ability index; and job satisfaction factors (planning issues, general attitudes, performance issues, management issues, supervisory issues, training and salary issues, and benefits issues). Regarding medical examination by an OM specialist, a plenty of data on health status as well as work ability, will be collected at enrolment phase, and during follow-up.

\section{Mental Health}

\section{O3E.2 RISK OF MENTAL HEALTH DISORDERS IN HUMAN SERVICE OCCUPATIONS: A REGISTER BASED STUDY OF 445,651 NORWEGIANS}

Petter Kristensen*, Therese N Hanvold, Rachel L Hasting, Suzanne L Merkus, Ingrid S Mehlum. National Institute of Occupational Health, Oslo, Norway

\subsection{6/OEM-2019-EPI.80}

Objectives Mental disorders contribute strongly to disability. Work in human service occupations has been considered a risk factor in several studies. We aimed at quantifying this relationship in a prospective follow-up of all employed persons born in Norway, 1967-1976.

Methods We conducted follow-up in several national registries. Based upon the ISCO98 four-digit code, we classified 2007 occupations into customer contact, client/patient contact, and reference (no contact). Client/patient contact was subdivided into health care, education and social work. We collected mental disorder diagnoses (ICD-10 F00-F99), in particular affective (F30-F39) and stress-related (F40-F48), in the Norwegian Patient Registry, 2008-2011. Four-year prevalence differences (PD) per 100 across occupational categories were estimated in binomial regression models adjusted for year of birth, marital history, current family pattern, and education level.

Results Among 445651 employed persons, 18\% held customer-related occupations while $25 \%$ held occupations with client/patient contact. The total four-year prevalence of mental disorder was 8.6/100 (38,207 patients). Affective and stress-related prevalences were 5.3 and 6.5 for women, and 3.0 and 3.2 for men, respectively. Adjusted PD estimates showed positive associations between client/patient contact occupations and mental disorders: for women, affective and stress-related disorder PD estimates were 1.1 (95\% confidence interval $(\mathrm{CI}) ; 0.9-1.3)$ and $1.3(\mathrm{CI} ; 1.0-1.5)$, respectively. The corresponding PD estimates for men were 1.7 (CI; 1.5-2.0) and 1.5 (CI; 1.2-1.7). We found strongest associations for women in social work (PD 2.3 for stress-related disorders) and men in health care (PD 2.6 for affective disorders). Associations with other mental disorder categories were weak, as were associations with customer contact occupations.

Conclusions Affective and stress-related morbidity was clearly associated with occupations involving client/patient contact for both sexes. We cannot dismiss health selection as an alternative to a causal effect of work-related factors. This registerbased study is not well suited to provide mechanistic explanations.

\section{E.3 CHANGES IN JOB SECURITY AND MENTAL HEALTH: AN ANALYSIS OF 14 ANNUAL WAVES OF AN AUSTRALIAN WORKING POPULATION PANEL SURVEY}

${ }^{1}$ Anthony LaMontagne*, ${ }^{1}$ Lan Say Too, ${ }^{2}$ Laura Punnett, ${ }^{3}$ Allison Milner. ${ }^{1}$ Centre for Population Health Research, Deakin University, Burwood, Australia; '2University of Massachussetts at Lowell, Lowell, USA; ${ }^{3}$ Melbourne School of Population and Global Health, Melbourne, Australia

\subsection{6/OEM-2019-EPI.81}

Background There is increasing recognition of job insecurity as an emerging issue in public health. We sought to examine whether job security improvements were associated with improvements in mental health in a large, working population-representative repeated-measures panel study.

Methods We used both within-person fixed effects (FE) and random effects (RE) regression to analyse data from 14 annual waves of a national Australian survey (19 169 persons, 106942 observations). Mental Health Inventory-5 scores (outcome) were modeled in relation to self-reported job security (categorical, quintiles), adjusting for age, year, education, and job change in the past year.

Results Both FE and RE models showed stepwise improvements in MHI-5 scores with improving job security, with stronger exposure-outcome relationships in the RE models, and for men compared to women. All models showed roughly monotonic improvements in MHI-5 score by quintile of improvement in job security. The strongest relationship was observed in the RE model for males: for a one-quintile improvement in job security, beta $=2.06$ [1.67, 2.46], and the following for two- (3.94 [3.54, 4.34]), three- (5.82 [5.40, $6.24])$, and four- $(7.18[6.71,7.64])$ quintile improvements. The FE model for males produced slightly smaller coefficients, reaching a maximum of $5.55[5.06,6.05]$ for a four-quintile improvement.

Conclusions This Australian national panel study showed a strong dose-response relationship between job security and depression and anxiety symptoms. Stronger causal inference over previous observational research is supported by the doseresponse finding and the relative consistency of the $\mathrm{FE}$ and RE results. Policy and practice intervention to improve job security could benefit population health. 